\title{
Simulation Embedded in Optimization - A Key for the Effective Learning Process in (About) Complex, Dynamical Systems
}

\author{
Elżbieta Kasperska and Elwira Mateja-Losa \\ Institute of Mathematics, Silesian University of Technology, Kaszubska 23, \\ 44-100 Gliwice, Poland \\ $\{$ e.kasperska, e.mateja\}@polsl.pl
}

\begin{abstract}
The purpose of this paper is to present some results of the experiments of type simulation embedded in optimization (on model type Systems Dynamics). They allow, not only, the so called, direct optimization, but extended sensitivity analysis of parameters and structures too. Authors have used languages Cosmic and Cosmos to support the learning process in (about) modelled dynamical system.
\end{abstract}

\section{Introduction}

We have problems understanding complex systems not only because they are rich in feedbacks $[2,3,4,5,6,7$, but because these complex systems are changing their structure while we are trying to understand them using a fixed structure approach.

System Dynamics was developed in the late 1950's and early 1960's at the Massachusetts Institute of Technology's Sloan School of Management by Jay W. Forrester.

The classical concept of System Dynamics assumes, that, during the time horizon of the model run or the simulation, the structure (given a-priori) will remain constant.

During last couple of years, some ideas of structural evolution have occurred in System Dynamics modelling and simulation. Firstly Prof. Coyle took the problem of, so called, "simulation during optimization". The question was: how to "optimize" the structure in order to achieve the desired behaviour? He performed experiments, using simulation language COSMIC and COSMOS 1]. It is a software tool that automatically links dynamics simulation model to an optimization package. This facility makes it possible to apply powerful optimization techniques to:

- fine tuning of policies in the model (DIRECT OPTIMIZATION),

- sensitivity analysis of the model (BASE VECTOR ANALYSIS),

- simplification of the structure of the model (SIMPLIFICATION),

- exploring the effects of forecasting and forward planning in the model (PLANNING HORIZON). 
In this paper some of such experiments are presented and on this background the context of leaning process is considered.

\section{Simulation Embedded in Optimization - Experiments on Model DYNBALANCE(3-1-III)}

The model named DYNBALANCE(3-1-III), created by Kasperska, was chosen like the object of experiments type simulation embedded in optimization. The figure 1 presents the general structure of this model. The graphical convention is adapted from Forrester [4. The main attention should be pay to the objective functions: "fob", "mmaxfo", which model the deciders preferences about of the behaviour of system, like the results of the influence of the decisions about the production, storing and selling. Below authors present the main results of some experiments, type simulation embedded in optimization.

\section{Experiment 1}

The objective function represents the profit from selling minus the total cost of production and the penalty factor connected with cost of the storage of product.

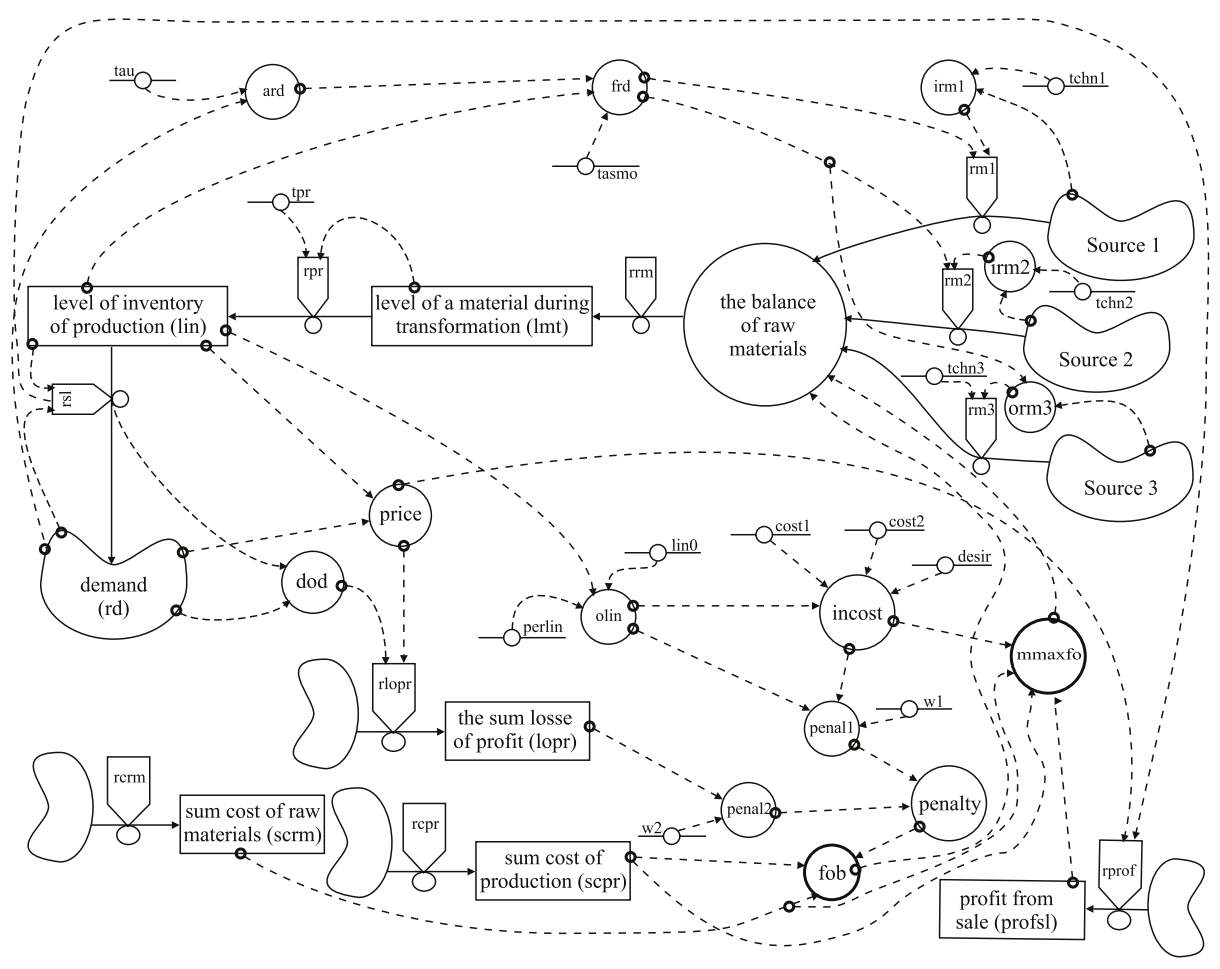

Fig. 1. Structure of model DYNBALANCE(3-1-III) 
The demand for product has "ramp" characteristic. Table 1 shows the results of the experiment.

Table 1. The main results of experiment 1

\begin{tabular}{lcccc}
\hline Parameter & Final value & Original value & Lower limit & Upper limit \\
\hline tchn1 & 0.000 & 20 & 0 & 40 \\
tchn2 & 11.332 & 10 & 0 & 40 \\
tchn3 & 39.984 & 20 & 0 & 40 \\
\hline Initial value of mmaxfo: & \multicolumn{1}{c}{$0.20233 E+07$} \\
Final value of mmaxfo: & $0.47194 E+07$ \\
Final value of inventory: & 221 \\
Final value of cost of production: & $24.295 E+05$ \\
Final value of price: & 50 \\
Final value of penalty (maxpen): & $0.040792 E+07$ \\
\hline
\end{tabular}

The conclusion from analysing the results of experiment 1, are as follows:

- the zero value of parameter "tchn1" implies the necessity of cutting off the production from 1st raw material (it seems that first technology is not economical, under the simulation condition about the price and the unit cost and the supplying from raw materials,

- the value of parameter "tchn2" is closed to its original value and "tchn3" is nearly equal to the upper limit - as can be seen the optimization process select such values that suits the objective function in the whole horizon of simulation,

- the final value of penalty for "mmaxfo" is rather small, comparing with the final value of profit - it seems that taken politics of production was really good.

\section{Experiment 2}

In this experiment the variable "fob" was chosen, like the objective function and we are looking for a minimum of it. Like the so called "active parameters" we have chosen: tchn1, tchn2, tchn3 and like the "base-parameter candidate" parameters: tan, tasmo, ucpr1, ucpr2, ucpr3, ucr1,ucr2, ucr3.

The initial value of objective function "fob" was: $0.29128 E+08$, and the final value of "fob" was: $0.25939 E+08$. The conclusion from analysing the results of experiment 1 , are as follows:

- many of parameters (for example: tan, ucr2, ucr3, ucpr1, ucpr2, ucpr3, tasmo) have kept their original value - that seems that their initial values were chosen reasonably good,

- the parameters: tchn1, tchn2, tchn3 (which were active in optimization dia$\log$ ) have changed their values considerably - it means that they are sensitive parameters of model, 
- this type of experiment gives more information about which parameters have more effect on improving the objective function.

\section{$3 \quad$ Final Remarks and Conclusions}

The purpose of the paper was to present some results of experiments of type simulation embedded in optimization on model type System Dynamics. These results show how the process of learning, in (about) complex, dynamical system, can become effective and supportive for both: deciders and us as modelers, as well. In "narrow" concept we can treat the modelling like a element of the global learning (see: three loops of learning by Radzicki, Sterman [6, 17) but in the other side modelling is a way of learning (specially, if we consider "self - made models").

Final conclusions are as follows:

- simulation embedded in optimization can become the effective, supportive way of learning about complex, dynamical system,

- both sensitivity analysis (parameters and structures give us the key information about elements and theirs connections (which determine the dynamical behaviour of system).

\section{References}

1. Coyle, R.G. (ed.): Cosmic and Cosmos. User manuals. The Cosmic Holding CO, London (1994)

2. Coyle, R.G.: System Dynamics Modelling. A Practical Approach. Chapman \& Hall, London (1996)

3. Coyle, R.G.: Simulation by repeated optimisation. J. Operat. Res. Soc. 50 (1999) 429-438

4. Forrester, J.W.: Industrial Dynamics. MIT Press, Massachusetts (1961)

5. Kasperska, E., Mateja-Losa, E., Słota, D.: Some dynamic balance of production via optimization and simulation within System Dynamics method. In: Hines, J.H., Diker, V.G. (eds.): Proc. 19th Int. Conf. of the System Dynamics Society. SDS, Atlanta (2001) 1-18

6. Radzicki, H.J.: Mr Hamilton, Mr Forrester and the foundation for evolutionary economics. In: Davidsen, P.I., Mollona, E. (eds.): Proc. 21st Int. Conf. of the System Dynamics Society. SDS, New York (2003) 1-41

7. Sterman, J.D.: Business dynamics - system thinking and modeling for a complex world. Mc Graw-Hill, Boston (2000) 